\title{
Optimal Pricing and Advertising Decisions in a Dynamic Multi- Channel Supply Chain
}

\author{
Yang Bai ${ }^{1}$, Byungjoo Park ${ }^{2}$ and Byeong-Yun Chang ${ }^{1, *}$ \\ ${ }^{1}$ School of Business, Ajou University, Suwon, Republic of Korea \\ ${ }^{2}$ School of Multimedia, Hannam University \\ b198716y@ajou.ac.kr,bjpark@hnu.kr,*bychang@ajou.ac.kr
}

\begin{abstract}
Channel coordination and advertising promotion strategy play very important roles in multi-channel supply chains. In our research, we consider the optimal pricing and advertising decisions for a manufacturer directing both traditional retailer channel and online channel, where advertising strategy contributes to accumulating goodwill. We studied Feedback Stackelberg Equilibria in a multi-channel under non-coop, unilateral coop, and bilateral coop advertising strategy scenarios. Among the three scenarios, the optimal wholesale price, online channel price, retail channel price and advertising strategies are obtained. The results show that a bilateral coop advertising promotion strategy always leads to a Pareto improving, and the manufacturer could use it to stimulate and influence the retailer's advertising decisions and thus ultimately promotes the whole channel's sales.
\end{abstract}

Keywords: Supply chain, Feedback Stackelberg equilibria, Game theory, Cooperative advertising, Online channel, Retailer channel

\section{Introduction}

In supply-chain management and marketing, pricing and optimal advertising decisions play important roles in today's business world. Manufacturers, according to the cooperative advertising promotion strategy, share costs with retailers, affect sales and ultimately improve total channel profits. For example, a manufacturer reimburses part of his retailer's advertising cost to stimulate and influence the retailer's advertising decisions, which ultimately promotes the manufacturer's sales.

Recently, more and more manufacturers have been using such a cooperative advertising promotion strategy, and as much as $40 \%$ of local advertising based on a coopadvertising program [1]. According to the Statistical Abstracts of the United States and various researchers, cooperative advertising expenditure in the U.S. grew to $\$ 36$ billion by 2015 , up from $\$ 25$ billion in 2007 [2, 3].

Nowadays, with the fast development of science and technology, e-business has become more and more important to consumer-goods supply chains. For instance, online retailer sales grew to 176 billion in 2010, up from 130 billion in 2008 [4]. As a result, ecommerce has stimulated many manufacturers to add online channels by engaging in direct sales.

Although various studies have been conducted on the cooperative advertising strategy or multi-channel integration strategy in past decades [4-8], very little research has considered pricing and advertising decisions in the manufacturer directing traditional retailer channel and online channel. The main contributions of this paper will be to enrich

Received (June 23, 2018), Review Result (September 4, 2018), Accepted (September 15, 2018)

* Corresponding Author 
the supply-chain pricing literature by including advertising decisions of manufacturers who are directing both traditional retailer channels and online channels based on goodwill.

This paper addresses the following research questions:

1. What is the optimal pricing policy of an online channel and a traditional channel?

2. How does the cooperative advertising promotion strategy affect a consumergoods supply chain.?

3. Does cooperative advertising improve channel profits and reduce conflict?

The rest of this paper is organized as follows. In section 1, we survey the pertinent literature and Section 2 develops the differential advertising and pricing compete models based on goodwill. Section 3 characterizes Stackelberg feedback equilibrium. Section 4 compares the main results derived from the three scenarios. The last section offers our conclusions and future research.

\section{Literature Review}

Numerous contributions made on cooperative advertising have focused on two categories, static game framework and dynamic game framework. Berger [6] first used a static game model for cooperative advertising where manufacturers direct retailers. B ased on Berger [6], most research studies the effect of an advertising allowance to the retailers offered by manufacturers [9-13]. They concluded that the cooperative advertising promotion program has a positive effect on the profits of the channel members.

Nerlove and Arrow [5] first developed the time-dependent advertising model. Chintagunta and Jain [7] extended the classical N-A model and addressed a manufacturerretailer, two-member marketing channel. They compared uncoordinated strategies and found that the total channel profits are higher when members follow the advertising promotion strategy. Jørgensen, Sigué and Zaccour [1] addressed a dynamic cooperative advertising strategy where a manufacturer directs a retailer's Stackelberg Equilibrium. Jørgensen, Sigué and Zaccour [1] showed that manufacturer and retailer control their long-term as well as short-term advertising efforts to improve their sales and consumer goodwill. They showed that support programs are much better than ones without support. Jørgensen, Sigué and Zaccour [8] extended their research in [1] to introduce decreased marginal returns to goodwill and designed two types of stationary feedback equilibria. Jørgensen, Taboubi, and Zaccour [14] studied the case where the retailer's promotions have negative effects on the brand image. Karray and Zaccour [15] considered the cooperative advertising strategy in a two-member marketing channel. As a result, cooperative support of the retailer could help the manufacturer mitigate the negative effect even when the retailer sells a private label. Giovanni and Roselli [17] found that under a revenue-sharing contract, a cooperative advertising strategy could mitigate the conflicts and always lead to Pareto improving.

Although all of above studies are based on one manufacture and one retailer, little of the literature has focused on competitive models [16] and even fewer on pricing and advertising decisions [17] with a manufacturer directing a retailer supply chain as well. In our paper, we modify the model addressed in Giovanni and Roselli [17] and Kim and Staelin [11]. Our proposal is to see if a cooperative support strategy has a positive effect on a manufacturer's directing a newly added online channel that is competitive with a traditional channel.

\section{Methods}

We suppose there are two marketing channels. One is a traditional channel, consisting of a manufacturer and its traditional retailer, and the other is a manufacturer's newly added online channel; the manufacturer sells its products through these two independent 
and competitive channels. The manufacturer of player $\mathrm{M}$ decides the wholesale price $\omega$ for the traditional retailer. It also chooses the subsidy rate, $0 \leq \eta_{M} \leq 1$, to support the retailer's advertising expenditure. Meanwhile, the retailer of player $\mathrm{R}$ chooses the subsidy rate, $0 \leq \eta_{\mathrm{R}} \leq 1$, to support the manufacturer's advertising expenditure. Each channel member's advertising efforts contribute to the accumulation of goodwill, $G(t)$. To capture the cooperative advertising strategy clearly, we extended the Nerlove and Arrow [5] dynamic advertising model according to the dynamics:

$$
\dot{G}(t)=\gamma A_{M}(t)+\epsilon A_{R}(t)-\delta G(t), \quad G(0)=G_{0} \geq 0 .
$$

$A_{M}(t)$ is the advertising efforts of the manufacturer, $A_{R}(t)$ is the advertising efforts of the retailer, $\gamma \geq 0$ and $\epsilon \geq 0$ are the advertising effectiveness, which means the marginal contributions of advertising efforts, $\delta \geq 0$ is the decay rate of the stock of the goodwill. Following Giovanni and Roselli [17], Kim and Staelin [11], any instant of time demand depends on price, and stock goodwill can be extended as follows:

$$
\begin{aligned}
& D_{1}\left(p_{1}(t), p_{2}(t), G(t)\right)=\theta \mu \sqrt{G}-b_{1} p_{1}+\beta p_{2}, \\
& D_{2}\left(p_{1}(t), p_{2}(t), G(t)\right)=(1-\theta) \mu \sqrt{G}-b_{2} p_{2}+\beta p_{1} .
\end{aligned}
$$

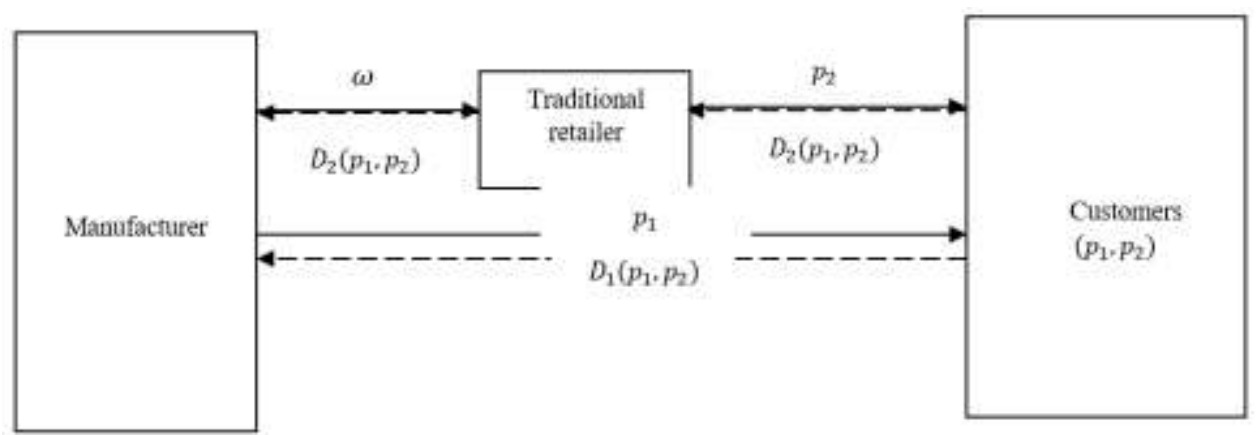

Figure 1. The Multi-Channel Distribution Diagram

In equations (2) and (3), $D_{1}$ and $D_{2}$ denote the demands of manufacturer who directs the online channel and the retail channel; $b_{1}, b_{2}$ and $\beta$ are nonnegative price elastic coefficients; $\mu>0$ represents the effectiveness of goodwill in current sales; and $0 \leq \theta \leq$ 1 represents the percentage of accumulated goodwill going to the channel members. The advertising costs are assumed to be convex and increasing that take the quadratic form:

$$
C\left(A_{M}(t)\right)=\frac{1}{2} A_{M}^{2}(t), C\left(A_{R}(t)\right)=\frac{1}{2} A_{R}^{2}(t) .
$$

We denote the channel members' use of $r \geq 0$ to be the discount rate. The manufacturer's objective function under an assumed infinite-time horizon is:

$$
\begin{aligned}
J_{M}=\operatorname{Max} \int_{0}^{\infty} & e^{-r t}\left\{\left(p_{1}(t)-c\right) D_{1}(t)+(\omega(t)-c) D_{2}(t)-\frac{\left(1-\eta_{R}(t)\right)}{2} A_{M}^{2}(t)\right. \\
& \left.-\frac{\eta_{M}}{2} A_{R}^{2}(t)\right\} d t .
\end{aligned}
$$

and the retailer is 


$$
\begin{gathered}
J_{R}=\operatorname{Max} \int_{0}^{\infty} e^{-r t}\left\{\left(p_{2}(t)-\omega(t)\right) D_{2}(t)-\frac{\left(1-\eta_{M}(t)\right)}{2} A_{R}^{2}((t)\right. \\
\left.-\frac{\eta_{R}(t)}{2} A_{M}^{2}(t)\right\} d t .
\end{gathered}
$$

\section{Feedback Stackelberg Equilibria}

\subsection{Feedback Equilibria without Advertising Support}

In this scenario, we assume each channel player decides on his advertising strategy and aims to maximize his benefits independently. Here, the manufacturer does not subsidy the retailer's advertising expenditure, and there is no coordination between them. Under an infinite-time horizon, the players' advertising strategies have a positive effect on building goodwill, which increases both players' sales and payoffs. In this case, we use a very simple price transaction model that is widely used nowadays in marketing research [4][21]. In order to avoid confusion, we omitted the time argument and assume $\mu=1$, $\beta=1$, as in [4]; when $b>\beta=1$, it means the traditional and online channel sale are identical products, and, the self-price has a greater effect than others], $b_{1}=b_{2}=b, c=$ 0 . Proposition 1 characterizes our main results for the non-coordinate equilibrium strategies.

Proposition 1. The non-coop scenario of the feedback Stackelberg Equilibrium wholesale price, online channel price, retail channel price, and advertising decisions are given by:

$$
\begin{aligned}
& \omega^{N}=\frac{b(1-\theta) \sqrt{G^{N}}+\theta \sqrt{G^{N}}}{2 b^{2}-2} \\
& p_{1}^{N}=\frac{b \theta \sqrt{G^{N}}+(1-\theta) \sqrt{G^{N}}}{2 b^{2}-2}, \\
& p_{2}^{N}=\frac{(1-\theta) \sqrt{G^{N}}\left(3 b^{2}-1\right)+2 b \theta \sqrt{G^{N}}}{4 b\left(b^{2}-1\right)}, \\
& A_{M}^{N}=\frac{\gamma\left(1+b^{2}-2 \theta+4 b \theta-2 b^{2} \theta+\theta^{2}-4 b \theta^{2}+3 b^{2} \theta^{2}\right)}{8\left(-b+b^{3}\right)(r+\delta)}, \\
& A_{R}^{N}=\frac{\epsilon(-1+\theta)^{2}}{16 b(r+\delta)} .
\end{aligned}
$$

Proposition 1 shows that optimal wholesale, online channel price, and traditional retailer price are goodwill-state-dependent, and the advertising strategies are constant. The results above are in accordance with Giovanni and Roselli [17] and Taboubi and Zaccour [18].

Proofs are given in Appendix 1.

Corollary 1. The optimal wholesale, online channel price, and traditional retailer price are goodwill-state-dependent; there is a positive relationship between goodwill level and channel pricing strategies.

$\frac{\partial \omega^{N}}{\partial G^{N}}=\frac{\theta+b(1-\theta)}{2\left(2 b^{2}-2\right) \sqrt{G^{N}}} \geq 0$, 
$\frac{\partial p_{1}^{N}}{\partial G^{N}}=\frac{b \theta+(1-\theta)}{2\left(2 b^{2}-2\right) \sqrt{G^{N}}} \geq 0$,

$\frac{\partial p_{2}^{N}}{\partial G^{N}}=\frac{(1-\theta)\left(3 b^{2}-1\right)+2 b \theta}{8 b\left(b^{2}-1\right) \sqrt{G^{N}}} \geq 0$.

The above corollary addresses a positive relationship between pricing strategies and goodwill.

Corollary 2. As price sensitivity increases, the optimal wholesale, online channel price, and traditional retailer price all decrease.

$\frac{\partial \omega^{N}}{\partial b}=\frac{-g+g \theta}{2\left(-1+b^{2}\right)}+\frac{b(-b g-g \theta+b g \theta)}{\left(-1+b^{2}\right)^{2}}<0 ，$

$\frac{\partial p_{1}^{N}}{\partial b}=\frac{g \theta}{2\left(-1+b^{2}\right)}+\frac{b(-g+g \theta-b g \theta)}{\left(-1+b^{2}\right)^{2}}<0$ ，

$\frac{\partial p_{2}^{N}}{\partial b}=\frac{[6 b(1-\theta)+2 \theta]-\left[8\left(b^{2}-1\right) \sqrt{G^{N}}+16 b^{2} \sqrt{G^{N}}\right]}{\left[8 b\left(b^{2}-1\right) \sqrt{G^{N}}\right]^{2}}<0$.

The above result is in line with Chutani and Sethi [19], showing that as b increases, the optimal wholesale, online channel price, and traditional retailer price all decrease.

Following Giovanni [20], we can get the accumulation of goodwill under a nonadvertising promotion strategy scenario by inserting (10) and (11) into equation (1):

$G_{\infty}^{N}=\frac{\gamma^{2}\left(1+b^{2}-2 \theta+4 b \theta-2 b^{2} \theta+\theta^{2}-4 b \theta^{2}+3 b^{2} \theta^{2}\right)}{8 \delta\left(-b+b^{3}\right)(r+\delta)}+\frac{\epsilon^{2}(1-\theta)^{2}}{16 b \delta(r+\delta)}$.

\subsection{Feedback Equilibria with Unilateral Advertising Support}

In this scenario, we consider the unilateral advertising promotion strategy. It means that only manufacturer reimburses the part of his retailer's advertising cost $\left(\eta_{M} \neq 0\right)$, to stimulate and influence the retailer's advertising decisions. Proposition 2 characterizes our main results for the coordinate equilibrium strategies.

Proposition 2. The unilateral coop scenario of the feedback Stackelberg Equilibrium wholesale price, online channel price, retail channel price, and advertising decisions are given by

$$
\begin{aligned}
& \omega^{C}=\frac{b(1-\theta) \sqrt{G^{C}}+\theta \sqrt{G^{C}}}{2 b^{2}-2}, \\
& p_{1}^{C}=\frac{b \theta \sqrt{G^{C}}+(1-\theta) \sqrt{G^{C}}}{2 b^{2}-2}, \\
& p_{2}^{C}=\frac{(1-\theta) \sqrt{G^{C}}\left(3 b^{2}-1\right)+2 b \theta \sqrt{G^{C}}}{4 b\left(b^{2}-1\right)}, \\
& A_{M}^{C}=\frac{\gamma\left(1+b^{2}-2 \theta+4 b \theta-2 b^{2} \theta+\theta^{2}-4 b \theta^{2}+3 b^{2} \theta^{2}\right)}{8\left(-b+b^{3}\right)(r+\delta)}, \\
& A_{R}^{C}=\frac{\epsilon\left(1+b^{2}-2 \theta+4 b \theta-2 b^{2} \theta+\theta^{2}-4 b \theta^{2}+3 b^{2} \theta^{2}\right)}{8\left(-b+b^{3}\right)(r+\delta)}+\frac{\epsilon(-1+\theta)^{2}}{32 b(r+\delta)},
\end{aligned}
$$




$$
\eta_{M}^{C}=\frac{2 V_{M}^{C^{\prime}}-V_{R}^{C^{\prime}}}{2 V_{M}^{C^{\prime}}+V_{R}^{C^{\prime}}}=\frac{5+3 b^{2}-10 \theta+16 b \theta-6 b^{2} \theta+5 \theta^{2}-16 b \theta^{2}+11 b^{2} \theta^{2}}{3+5 b^{2}-6 \theta+16 b \theta-10 b^{2} \theta+3 \theta^{2}-16 b \theta^{2}+13 b^{2} \theta^{2}} .
$$

Proofs are given in Appendix 2.

The above results show us that as the goodwill increases, the optimal wholesale, online channel price, and traditional retailer price are increased, and the results also show us that the advertising strategies are state-independent under the coop advertising strategy as well. The result in equation (24) shows that the manufacturer will support the retailer's advertising strategy if and only if the manufacturer's margin is more than half of the retailer's. If the retailer margin is zero, the manufacturer will support all of the cost.

Corollary 3. The wholesale price and retail channel price decrease with the sharing parameter; the online channel price and advertising increase with the sharing parameter.

$$
\begin{aligned}
& \frac{\partial \omega^{C}}{\partial \theta}=\frac{\sqrt{G^{C}}-b \sqrt{G^{C}}}{2 b^{2}-2} \leq 0, \\
& \frac{\partial p_{1}^{C}}{\partial \theta}=\frac{b \sqrt{G^{C}}-\sqrt{G^{C}}}{2 b^{2}-2} \geq 0, \\
& \frac{\partial p_{2}^{C}}{\partial \theta}=\frac{-(3 b+1)(b-1) \sqrt{G^{C}}}{4 b\left(b^{2}-1\right)} \leq 0, \\
& \frac{\partial A_{M}^{C}}{\partial \theta}=\frac{\gamma\left(-2+4 b-2 b^{2}+2 \theta-8 b \theta+6 b^{2} \theta\right)}{\left(b^{3}-b\right)(r+\delta)} \geq 0, \text { if } \theta>\frac{b-1}{3 b-1}, \\
& \frac{\partial A_{R}^{C}}{\partial \theta}=\frac{\varepsilon\left(-6+16 b-10 b^{2}+6 \theta-32 b \theta+26 b^{2} \theta\right)}{32 b\left(b^{2}-1\right)(r+\delta)} \geq 0, \text { if } \theta>\frac{5 b-3}{13 b-3} .
\end{aligned}
$$

Because $\frac{5 b-3}{13 b-3}>\frac{b-1}{3 b-1}>0$, the results show that when the sharing parameter $\theta>$ $\frac{5 b-3}{13 b-3}$, as sharing parameter $\theta$ increases, the manufacturer supports the advertising strategy more, ultimately improving total accumulation of goodwill. Which also decreases the demand of the retailer. So, in order to increase his profits, he must decrease the price as well. Meanwhile, the manufacturer must increase the online channel price to reduce conflict in order to get to win-win.

Following Giovanni [20], we can get the accumulation of goodwill under a nonadvertising promotion strategy scenario by inserting (22) and (23) into equation (1):

$$
\begin{gathered}
G_{\infty}^{C}=\frac{\left(\gamma^{2}+\epsilon^{2}\right)\left(1+b^{2}-2 \theta+4 b \theta-2 b^{2} \theta+\theta^{2}-4 b \theta^{2}+3 b^{2} \theta^{2}\right)}{8 \delta\left(-b+b^{3}\right)(r+\delta)} \\
+\frac{\epsilon^{2}(1-\theta)^{2}}{32 b \delta(r+\delta)} .
\end{gathered}
$$

\subsection{Feedback Equilibria with Bilateral Advertising Support}

In this scenario, we consider the bilateral advertising promotion strategy. It means that the manufacturer and retailer reimburse the part of each other's advertising costs $\left(\eta_{M} \neq\right.$ $0, \eta_{R} \neq 0$,), to stimulate and influence the retailer's advertising decisions. Proposition 3 characterizes our main results for the bilateral advertising promotion strategy. 
Proposition 3. The bilateral coop scenario of the feedback Stackelberg Equilibrium wholesale price, online channel price, retail channel price, and advertising decisions are given by

$$
\begin{aligned}
& \omega^{B C}=\frac{b(1-\theta) \sqrt{G^{B C}}+\theta \sqrt{G^{B C}}}{2 b^{2}-2}, \\
& p_{1}^{B C}=\frac{b \theta \sqrt{G^{B C}}+(1-\theta) \sqrt{G^{B C}}}{2 b^{2}-2}, \\
& p_{2}^{B C}=\frac{(1-\theta)\left(3 b^{2}-1\right) \sqrt{G^{B C}}+2 b \theta \sqrt{G^{B C}}}{4 b\left(b^{2}-1\right)}, \\
& A_{R}^{B C}=\frac{\epsilon(-1+\theta)^{2}}{16 b(r+\delta)}+\frac{\epsilon\left(1+b^{2}(1-\theta)^{2}+4 b \theta(1-\theta)+2 \theta\left(b^{2}-1\right)+\theta^{2}\right)}{4 b\left(b^{2}-1\right)(r+\delta)}, \\
& A_{M}^{B C}=\frac{\gamma(-1+\theta)^{2}}{16 b(r+\delta)}, \\
& \eta_{M}^{B C}=\frac{5+3 b^{2}-10 \theta+16 b \theta-6 b^{2} \theta+5 \theta^{2}-16 b \theta^{2}+11 b^{2} \theta^{2}}{3+5 b^{2}-6 \theta+16 b \theta-10 b^{2} \theta+3 \theta^{2}-16 b \theta^{2}+13 b^{2} \theta^{2}} \\
& \eta_{R}^{B C}=\frac{2\left(-1+b^{2}\right)(-1+\theta)^{2}}{3+5 b^{2}-6 \theta+16 b \theta-10 b^{2} \theta+3 \theta^{2}-16 b \theta^{2}+13 b^{2} \theta^{2}} .
\end{aligned}
$$

Proofs are given in Appendix 3.

As in the previous scenarios, the optimal wholesale, online channel price, and traditional retailer price are goodwill-state-dependent, and the advertising strategies are constant. The above results also show us that the manufacturer will support the retailer's advertising strategy if and only if the manufacturer's margin is more than half of the retailer's. If the retailer margin is zero, the manufacturer will support all of the cost. Meanwhile, if the retailer's margin is not zero, he will always support the manufacturer's advertising strategy.

Following Giovanni [20], we can get the accumulation of goodwill under a nonadvertising promotion strategy scenario by inserting (34) and (35) into equation (1):

$$
\begin{gathered}
G_{\infty}^{B C}=\frac{\left(\gamma^{2}+\epsilon^{2}\right)\left(1+b^{2}-2 \theta+4 b \theta-2 b^{2} \theta+\theta^{2}-4 b \theta^{2}+3 b^{2} \theta^{2}\right)}{8 \delta\left(-b+b^{3}\right)(r+\delta)} \\
+\frac{\left(\gamma^{2}+\epsilon^{2}\right)(1-\theta)^{2}}{32 b \delta(r+\delta)} .
\end{gathered}
$$

\section{Comparison of Equilibrium}

In this section we compare the difference of the advertising strategies, outcomes, and accumulated goodwill of the non-coop, unilateral coop and bilateral coop scenarios in numerical examples. Our purpose is to check whether the cooperative advertising strategy improves channel profits and reduces the conflict, and then to identify the coop scenario of feedback Stackelberg Equilibrium, always leading to a Pareto improving.

In order to get the numerical results, we set the sharing parameter $\theta$ to vary from 0 to 0.99 , and $\gamma=0.3 ; \epsilon=0.3 ; \delta=0.1 ; r=0.1 ; b=1.1$ to run the simulation.

Figure 2 illustrates that as the market-sharing parameter $\theta$ increases to 1 , then the demand of the retailer will approach 0 ; so it is not meaningful for the retailer to investigate advertisement under the non-cooperative advertising strategy. But under the cooperative 
advertising strategies, the retailer could recoup his losses by the subsidy from the manufacturer, so he would like to investigate more than the non-cooperative advertising strategy. In addition, there is no difference between a scenario with unilateral coop and bilateral coop advertising strategy. It means:

$A_{R}^{B C}=A_{R}^{C}>A_{R}^{N}$

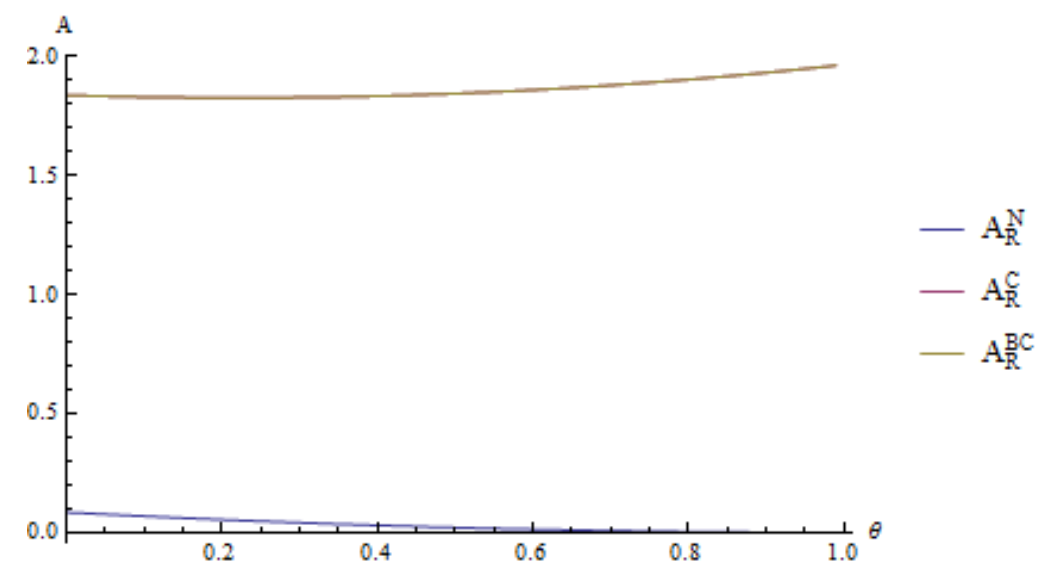

Figure 2. Relationship between Retailer's Advertising and the Market-Sharing Parameter $\boldsymbol{\theta}$

Figure 3 shows that the stock of goodwill under the bilateral cooperative advertising strategy is always higher than under the non-cooperative or under unilateral advertising strategy. It means:

$G_{\infty}^{B C}>G_{\infty}^{C}>G_{\infty}^{N}$

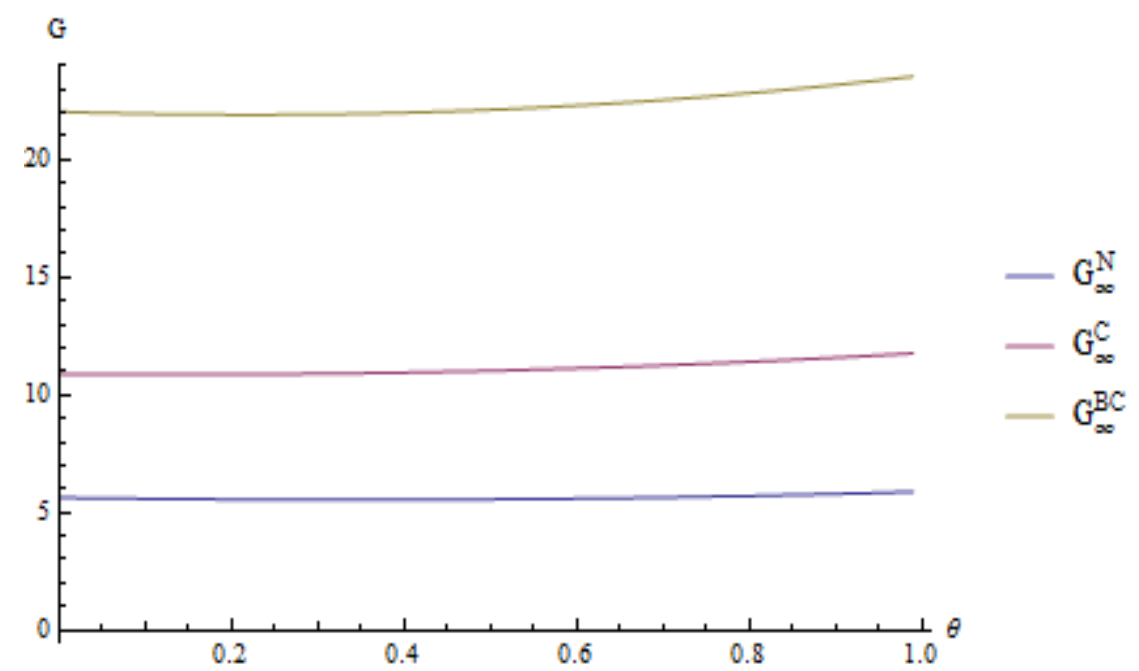

Figure 3. Relationship between Stock of Goodwill and Market-sharing Parameter $\theta$

Figure 4 illustrates that when the sharing parameter $\theta$ increases, the manufacturer supports the advertising strategy more, ultimately improving total accumulation of demand and profits. Figure 5 shows that; the sharing parameter increase also decreases the demand of the retailer. In other words, when the market-sharing parameter $\theta$ increases to 1 , then the demand of the retailer will approach 0 , which leads the profits of the retailer 
to be 0 . But as the manufacturer supports the advertising expenditure of the retailer, his profit is much higher than with the non-cooperative strategy, which means that in the coop advertising strategies, both channels are better off than with the non-coop advertising strategy. In addition, a bilateral cooperative advertising strategy is always higher than others. Thus, the bilateral cooperative advertising strategy could mitigate the conflict and always lead to a Pareto improving. Which means: $V_{M}^{B C}>V_{M}^{C}>$ $V_{M}^{N}$, and, $V_{R}^{B C}>V_{R}^{C}>V_{R}^{N}$

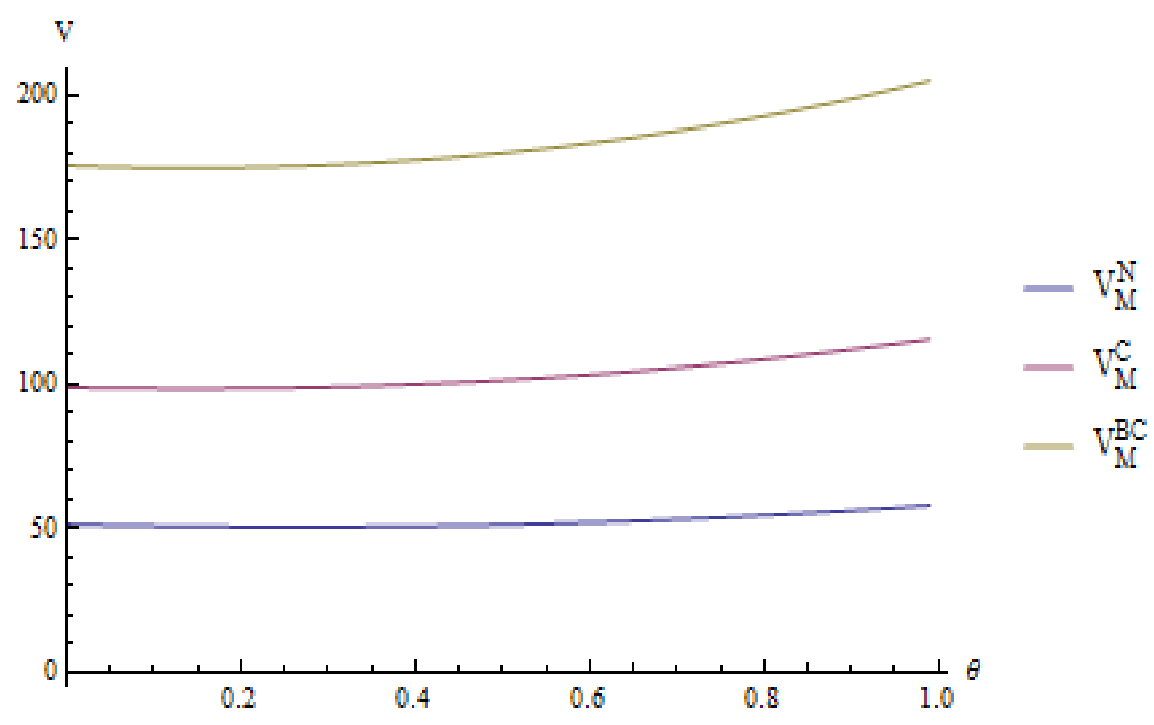

Figure 4. Relationship between Manufacturer's Profit and market-sharing Parameter $\theta$

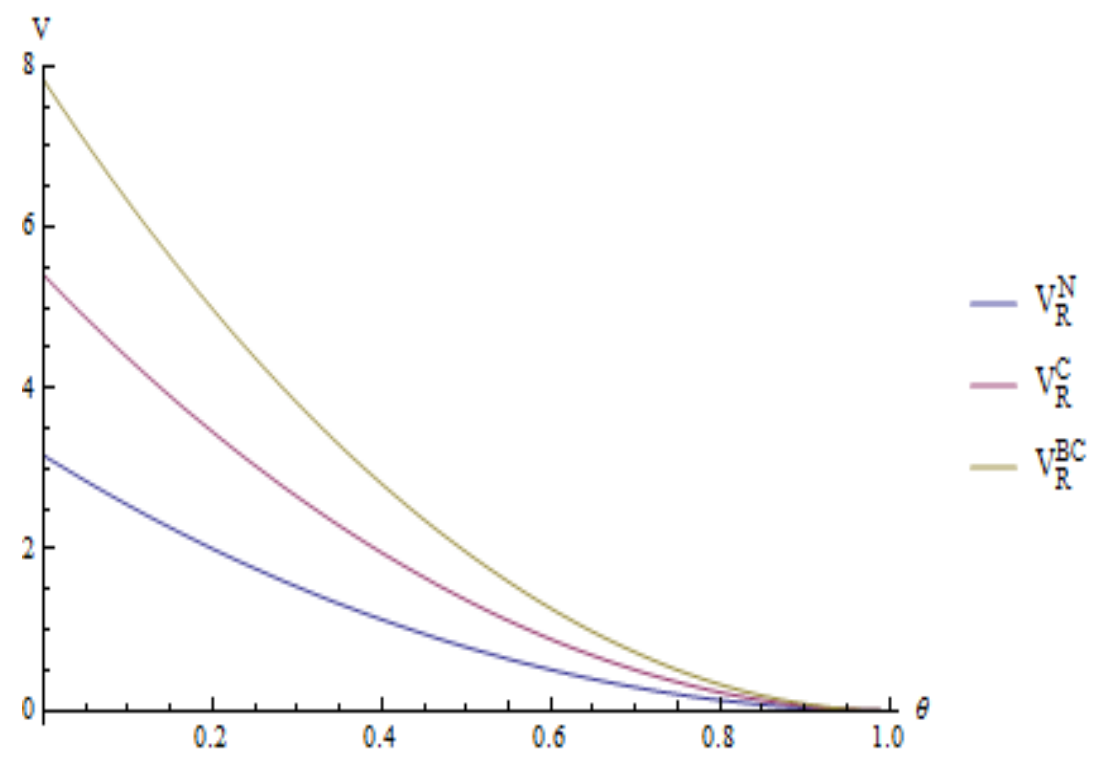

Figure 5. Relationship between Retailer's Profit and Market-Sharing Parameter $\theta$

\section{Conclusions}

We have characterized the Feedback Stackelberg Equilibria in a multi-channel under the non-coop, unilateral coop, and bilateral coop advertising strategy scenarios. One is a traditional channel formed by a manufacturer and its traditional retailer; the other is a 
manufacturer's newly added online channel. In our research, we compute the optimal wholesale price, online channel price, retail channel price, and advertising strategies. We obtain our main results as follows:

In all of the advertising strategy scenarios, the optimal wholesale, online channel price, and traditional retailer price are goodwill-state-dependent, and the advertising strategies are constant;

Offering an advertising support strategy represents a positive way to alleviate the conflict with the newly added online multi-channel, ultimately improve the profits of each channel member, which means that, the cooperative advertising strategies could mitigate the conflict and always lead to a Pareto improving.

Under the bilateral cooperative advertising strategy, it has significant effects than others.

Future research can be extended in several directions. First, we assumed the multichannel member's sale to be of identical products in our research, but retailers can also introduce a private label to the consumers. Second, quality improvement as operational tools can be also introduced to continue to build and accumulate goodwill. Finally, we used the linear value functions in our research. Further research can continue to extend our model to linear-quadratic differential games.

\section{Appendix 1}

We first need to establish the value functions $V_{M}^{N}(G), V_{R}^{N}(G)$ of the manufacturer with a newly added online channel and traditional retailer channel in a continuously differentiable dynamic framework, which for all $G^{N}(t)$ satisfies equation (1) and the HJB (Hamilton-Jacobi-Bellman) equations. We write the HJB equations for value functions in the non-coop scenario of two channels as follows:

$$
\begin{aligned}
& r V_{M}^{N}=\left\{p_{1}^{N}(\theta\right.\left.\sqrt{G^{N}}-b p_{1}^{N}+p_{2}^{N}\right)+\omega^{N}\left[(1-\theta) \sqrt{G^{N}}-b p_{2}^{N}+p_{1}^{N}\right]-\frac{1}{2}\left(A_{M}^{N}\right)^{2} \\
&\left.+V_{M}^{N^{\prime}}\left(\gamma A_{M}^{N}+\epsilon A_{R}^{N}-\delta G^{N}\right)\right\}, \\
& r V_{R}^{N}=\left\{\left(p_{2}^{N}-\omega^{N}\right)\left[(1-\theta) \sqrt{G^{N}}-b p_{2}^{N}+p_{1}^{N}\right]-\frac{1}{2}\left(A_{R}^{N}\right)^{2}\right. \\
&\left.+V_{R}^{N^{\prime}}\left(\gamma A_{M}^{N}+\epsilon A_{R}^{N}-\delta G^{N}\right)\right\} .
\end{aligned}
$$

Maximizing the first-order condition for $p_{2}^{N}$ and $A_{R}^{N}$ of the retailer gives:

$$
\begin{aligned}
& p_{2}^{N}=\frac{(1-\theta) \sqrt{G^{N}}+p_{1}^{N}+b \omega^{N}}{2 b}, \\
& A_{R}^{N}=V_{R}^{N^{\prime}} \epsilon .
\end{aligned}
$$

Substituting (41) into the manufacturer's HJB equations (39), we can obtain the functions as:

$$
\begin{array}{r}
r V_{M}^{N}=\max \left\{p_{1}^{N}\left(\theta \sqrt{G^{N}}-b p_{1}^{N}+\frac{(1-\theta) \sqrt{G^{N}}+p_{1}^{N}+b \omega^{N}}{2 b}\right)\right. \\
+\omega^{N}\left[(1-\theta) \sqrt{G^{N}}-b \frac{(1-\theta) \sqrt{G^{N}}+p_{1}^{N}+b \omega^{N}}{2 b}+p_{1}^{N}\right] \\
\left.-\frac{1}{2}\left(A_{M}^{N}\right)^{2}+V_{M}^{N \prime}\left(\gamma A_{M}^{N}+\epsilon A_{R}^{N}-\delta G^{N}\right)\right\} .
\end{array}
$$

By differentiating equation (43) on $p_{1}^{N}, \omega^{N}$ and $A_{M}^{N}$, we obtain 
$p_{1}^{N}=\frac{b \theta \sqrt{G^{N}}+(1-\theta) \sqrt{G^{N}}}{2 b^{2}-2}$,

$\omega^{N}=\frac{b(1-\theta) \sqrt{G^{N}}+\theta \sqrt{G^{N}}}{2 b^{2}-2}$,

$A_{M}^{N}=V_{M}^{N \prime} \gamma$

Then, substituting (44) and (45) into (41), we obtain

$p_{2}^{N}=\frac{(1-\theta) \sqrt{G^{N}}\left(3 b^{2}-1\right)+2 b \theta \sqrt{G^{N}}}{4 b\left(b^{2}-1\right)}$.

We look for the linear value functions,

$V_{M}^{N}=M_{1}^{N} G^{N}+M_{2}^{N}, V_{M}^{N \prime}=M_{1}^{N}$,

$V_{R}^{N}=R_{1}^{N} G^{N}+R_{2}^{N}, V_{R}^{N \prime}=R_{1}^{N}$.

where the parameters $M_{1}^{N}, M_{2}^{N}, R_{1}^{N}, R_{2}^{N}$, are the coefficients of the value function. Inserting (42) and (44)-(47) on the right-hand side of the HJB equations and simultaneous equations (48) and (49), we can obtain the following four equations:

$$
\begin{aligned}
& 2 b \theta\left(2 b^{2}-2\right)((1-\theta)+b \theta)-2 b^{2}((1-\theta)+b \theta)((1-\theta)+b \theta) \\
& +((1-\theta)+b \theta)\left((1-\theta)\left(3 b^{2}-1\right)+2 b \theta\right) \\
& +2 b\left(2 b^{2}-2\right)(b(1-\theta)+\theta)(1-\theta) \\
& -b(b(1-\theta)+\theta)\left((1-\theta)\left(3 b^{2}-1\right)+2 b \theta\right) \\
& +2 b(b(1-\theta)+\theta)(b \theta+(1-\theta)) \\
& =2 b\left(2 b^{2}-2\right)^{2}(r+\delta) M_{1}^{N} \text {, } \\
& \left(M_{1}^{N}\right)^{2} \gamma^{2}+2 M_{1}^{N} \epsilon^{2} R_{1}^{N}=2 r M_{2}^{N} \text {, } \\
& 2 b\left(2 b^{2}-2\right)(1-\theta)^{2}\left(b^{2}-1\right)-b\left((1-\theta)\left(3 b^{2}-1\right)+2 b \theta\right)(1-\theta)\left(b^{2}-1\right) \\
& +2 b((1-\theta)+b \theta)(1-\theta)\left(b^{2}-1\right) \\
& =4 b^{2}\left(2 b^{2}-2\right)^{2}(r+\delta) R_{1}^{N} \text {, } \\
& 2 R_{1}^{N} M_{1}^{N} \gamma^{2}+\left(R_{1}^{N}\right)^{2} \epsilon^{2}=2 r R_{2}^{N} .
\end{aligned}
$$

where the parameters $M_{1}^{N}, M_{2}^{N}, R_{1}^{N}, R_{2}^{N}$ can be easily obtained through simultaneous equations (50) - (53). Then, substituting $M_{1}^{N}$ and $R_{1}^{N}$ into (42) and (46), we obtain

$A_{M}^{N}=\frac{\gamma\left(1+b^{2}-2 \theta+4 b \theta-2 b^{2} \theta+\theta^{2}-4 b \theta^{2}+3 b^{2} \theta^{2}\right)}{8\left(-b+b^{3}\right)(r+\delta)}$,

$A_{R}^{N}=\frac{\epsilon(-1+\theta)^{2}}{16 b(r+\delta)}$.

\section{Appendix 2}

We need to establish the value functions $V_{M}^{C}(G), V_{R}^{C}(G)$ of the manufacturer with a newly added online channel and traditional retailer channel in a continuously differentiable dynamic framework, which for all $G^{C}(t)$ satisfies equation (1) and the HJB (Hamilton-Jacobi-Bellman) equations. We rewrite the HJB equations for value functions in the coop scenario of the multi-channel as follows: 


$$
\begin{gathered}
r V_{M}^{C}=\left\{p_{1}^{C}\left(\theta \sqrt{G^{C}}-b p_{1}^{C}+p_{2}^{C}\right)+\omega^{C}\left[(1-\theta) \sqrt{G^{C}}-b p_{2}^{C}+p_{1}^{C}\right]-\frac{1}{2}\left(A_{M}^{C}\right)^{2}\right. \\
\left.-\frac{\eta_{M}^{C}}{2}\left(A_{R}^{C}\right)^{2}+V_{M}^{C^{\prime}}\left(\gamma A_{M}^{C}+\epsilon A_{R}^{C}-\delta G^{C}\right)\right\}, \\
r V_{R}^{C}=\left\{\left(p_{2}^{C}-\omega^{C}\right)\left[(1-\theta) \sqrt{G^{C}}-b p_{2}^{C}+p_{1}^{C}\right]-\frac{\left(1-\eta_{M}^{C}\right)}{2}\left(A_{R}^{C}\right)^{2}\right. \\
\left.+V_{R}^{C^{\prime}}\left(\gamma A_{M}^{C}+\epsilon A_{R}^{C}-\delta G^{C}\right)\right\} .
\end{gathered}
$$

maximizing the first-order condition for $p_{2}^{C}$ and $A_{R}^{C}$ of the retailer as:

$$
\begin{aligned}
& p_{2}^{C}=\frac{(1-\theta) \sqrt{G^{C}}+p_{1}^{C}+b \omega^{C}}{2 b}, \\
& A_{R}^{C}=\frac{V_{R}^{C^{\prime}} \epsilon}{1-\eta_{M}^{C}} .
\end{aligned}
$$

Substituting (58) and (59) into the manufacturer's HJB equations (56), and by differentiating equation on $p_{1}^{C}, \omega^{C}, A_{M}^{C}$ and $\eta_{M}^{C}$, we can obtain:

$$
\begin{aligned}
& \omega^{C}=\frac{b(1-\theta) \sqrt{G^{C}}+\theta \sqrt{G^{C}}}{2 b^{2}-2}, \\
& p_{1}^{C}=\frac{b \theta \sqrt{G^{C}}+(1-\theta) \sqrt{G^{C}}}{2 b^{2}-2}, \\
& A_{M}^{C}=V_{M}^{C^{\prime}} \gamma, \\
& \eta_{M}^{C}=\frac{2 V_{M}^{C^{\prime}}-V_{R}^{C^{\prime}}}{2 V_{M}^{C^{\prime}}+V_{R}^{C^{\prime}}} .
\end{aligned}
$$

Then, substituting (60) and (61) into (58), we obtain

$$
p_{2}^{C}=\frac{(1-\theta) \sqrt{G^{C}}\left(3 b^{2}-1\right)+2 b \theta \sqrt{G^{C}}}{4 b\left(b^{2}-1\right)} \text {. }
$$

We look for the linear value functions,

$$
\begin{aligned}
& V_{M}^{C}=M_{1}^{C} G^{C}+M_{2}^{C}, V_{M}^{C \prime}=M_{1}^{C}, \\
& V_{R}^{C}=R_{1}^{C} G^{C}+R_{2}^{C}, V_{R}^{C \prime}=R_{1}^{C} .
\end{aligned}
$$

where the parameters $M_{1}^{C}, M_{2}^{C}, R_{1}^{C}, R_{2}^{C}$, are the coefficients of the value function. Inserting (59)-(64) on the right-hand side of the HJB equations and simultaneous equations (65) and (66), we can obtain the following four equations:

$$
\begin{aligned}
2 b \theta\left(2 b^{2}-2\right)( & (1-\theta)+b \theta)-2 b^{2}((1-\theta)+b \theta)((1-\theta)+b \theta) \\
& +((1-\theta)+b \theta)\left((1-\theta)\left(3 b^{2}-1\right)+2 b \theta\right) \\
& +2 b\left(2 b^{2}-2\right)(b(1-\theta)+\theta)(1-\theta) \\
& -b(b(1-\theta)+\theta)\left((1-\theta)\left(3 b^{2}-1\right)+2 b \theta\right) \\
& +2 b(b(1-\theta)+\theta)(b \theta+(1-\theta)) \\
& =2 b\left(2 b^{2}-2\right)^{2}(r+\delta) M_{1}^{C}
\end{aligned}
$$




$$
\begin{aligned}
& \begin{array}{l}
4\left(\gamma M_{1}^{C}\right)^{2}-\epsilon^{2}\left(2 M_{1}^{C}+R_{1}^{C}\right)\left(2 M_{1}^{C}-R_{1}^{C}\right)+4 M_{1}^{C} \epsilon^{2}\left(2 M_{1}^{C}+R_{1}^{C}\right)=8 r M_{2}^{C}, \\
2 b\left(2 b^{2}-2\right)(1-\theta)^{2}\left(b^{2}-1\right)-b\left((1-\theta)\left(3 b^{2}-1\right)+2 b \theta\right)(1-\theta)\left(b^{2}-1\right) \\
+2 b((1-\theta)+b \theta)(1-\theta)\left(b^{2}-1\right) \\
=4 b^{2}\left(2 b^{2}-2\right)^{2}(r+\delta) R_{1}^{C},
\end{array} \\
& R_{1}^{C} \epsilon^{2}\left(2 M_{1}^{C}+R_{1}^{C}\right)+4 M_{1}^{C} R_{1}^{C} \gamma^{2}=4 r R_{2}^{C}, .
\end{aligned}
$$

where the parameters $M_{1}^{C}, M_{2}^{C}, R_{1}^{C}, R_{2}^{C}$ can be easily obtained through simultaneous equations (67) - (70). Then, substituting $M_{1}^{C}$ and $R_{1}^{C}$ into (59),(62) and (63), we obtain

$$
\begin{gathered}
A_{M}^{C}=\frac{\gamma\left(1+b^{2}-2 \theta+4 b \theta-2 b^{2} \theta+\theta^{2}-4 b \theta^{2}+3 b^{2} \theta^{2}\right)}{8\left(-b+b^{3}\right)(r+\delta)}, \\
A_{R}^{C}=\frac{\epsilon\left(1+b^{2}-2 \theta+4 b \theta-2 b^{2} \theta+\theta^{2}-4 b \theta^{2}+3 b^{2} \theta^{2}\right)}{8\left(-b+b^{3}\right)(r+\delta)}+\frac{\epsilon(-1+\theta)^{2}}{32 b(r+\delta)}, \\
\eta_{M}^{C}=\frac{2 V_{M}^{C^{\prime}}-V_{R}^{C^{\prime}}}{2 V_{M}^{C^{\prime}}+V_{R}^{C^{\prime}}}=\frac{5+3 b^{2}-10 \theta+16 b \theta-6 b^{2} \theta+5 \theta^{2}-16 b \theta^{2}+11 b^{2} \theta^{2}}{3+5 b^{2}-6 \theta+16 b \theta-10 b^{2} \theta+3 \theta^{2}-16 b \theta^{2}+13 b^{2} \theta^{2}} .
\end{gathered}
$$

\section{Appendix 3}

We need to establish the value functions $V_{M}^{B C}(G), V_{R}^{B C}(G)$ of the manufacturer with a newly added online channel and traditional retailer channel in a continuously differentiable dynamic framework, which for all $G^{B C}(t)$ satisfies equation (1) and the HJB (Hamilton-Jacobi-Bellman) equations. We rewrite the HJB equations for value functions in the bilateral coop scenario of the multi-channel as follows:

$$
\begin{aligned}
r V_{M}^{B C}=\left\{p_{1}^{B C}(\right. & \left.\theta \sqrt{G^{B C}}-b p_{1}^{B C}+p_{2}^{B C}\right)+\omega^{B C}\left[(1-\theta) \sqrt{G^{B C}}-b p_{2}^{B C}+p_{1}^{B C}\right] \\
& -\frac{\left(1-\eta_{R}^{B C}\right)}{2}\left(A_{M}^{B C}\right)^{2}-\frac{\eta_{M}^{B C}}{2}\left(A_{R}^{B C}\right)^{2} \\
& \left.+V_{M}^{B C^{\prime}}\left(\gamma A_{M}^{B C}+\epsilon A_{R}^{B C}-\delta G^{B C}\right)\right\}, \\
r V_{R}^{B C}=\left\{\left(p_{2}^{B C}-\omega^{B C}\right)\left[(1-\theta) \sqrt{G^{B C}}-b p_{2}^{B C}+p_{1}^{B C}\right]-\frac{\left(1-\eta_{M}^{B C}\right)}{2}\left(A_{R}^{B C}\right)^{2}\right. & \left.-\frac{\eta_{R}^{B C}}{2}\left(A_{M}^{B C}\right)^{2}+V_{R}^{B C^{\prime}}\left(\gamma A_{M}^{B C}+\epsilon A_{R}^{B C}-\delta G^{B C}\right)\right\}
\end{aligned}
$$

Take the first order conditions respect to $p_{2}^{B C}, A_{R}^{B C}$ and $A_{M}^{B C}$ to maximization the retailer as:

$$
\begin{aligned}
p_{2}^{B C} & =\frac{(1-\theta) \sqrt{G^{B C}}+p_{1}^{B C}+b \omega^{B C}}{2 b}, \\
A_{R}^{B C} & =\frac{V_{R}^{B C^{\prime}} \gamma}{1-\eta_{M}^{B C}} . \\
A_{M}^{B C} & =\frac{V_{R}^{B C^{\prime}} \epsilon}{\eta_{R}^{B C}} .
\end{aligned}
$$

Substituting (76)-(78) into the manufacturer's HJB equations (74), we obtain: 


$$
\begin{array}{r}
r V_{M}^{B C}=\max \left\{p_{1}^{B C}\left(\theta \sqrt{G^{B C}}-b p_{1}^{B C}+\frac{(1-\theta) \sqrt{G^{B C}}+p_{1}^{B C}+b \omega^{B C}}{2 b}\right)\right. \\
+\omega^{B C}\left[(1-\theta) \sqrt{G^{B C}}-b \frac{(1-\theta) \sqrt{G^{B C}}+p_{1}^{B C}+b \omega^{B C}}{2 b}+p_{1}^{B C}\right] \\
\left.-\frac{\left(1-\eta_{R}^{B C}\right)}{2}\left(\frac{V_{R}^{B C^{\prime}} \epsilon}{\eta_{R}^{B C}}\right)^{2}-\frac{\eta_{M}^{B C}}{2}\left(\frac{V_{R}^{B C^{\prime}} \gamma}{1-\eta_{M}^{B C}}\right)^{2}+V_{M}^{B C^{\prime}}\left(\gamma \frac{V_{R}^{B C^{\prime}} \epsilon}{\eta_{R}^{B C}}+\epsilon \frac{V_{R}^{B C^{\prime}} \gamma}{1-\eta_{M}^{B C}}-\delta G^{B C}\right)\right\} .
\end{array}
$$

By the differential of equation (79) on $p_{1}^{B C}, \omega^{B C}, \eta_{R}^{B C}$ and $\eta_{M}^{B C}$, we obtain:

$p_{1}^{B C}=\frac{[b \theta+(1-\theta)] \sqrt{G^{B C}}}{2 b^{2}-2}$,

$\omega^{B C}=\frac{[b(1-\theta)+\theta] \sqrt{G^{B C}}}{2 b^{2}-2}$,

$\eta_{M}^{B C}=\frac{2 V_{M}^{B C^{\prime}}-V_{R}^{B C^{\prime}}}{2 V_{M}^{B C^{\prime}}+V_{R}^{B C^{\prime}}}$,

$\eta_{R}^{B C}=\frac{2 V_{R}^{B C^{\prime}}}{2 V_{M}^{B C^{\prime}}+V_{R}^{B C^{\prime}}}$.

Then, substituting (80) and (81) into (76), we obtain:

$p_{2}^{B C}=\frac{\left[(1-\theta)\left(3 b^{2}-1\right)+2 b \theta\right] \sqrt{G^{B C}}}{b\left(4 b^{2}-4\right)}$.

We looking for linear value functions,

$V_{M}^{B C}=M_{1}^{B C} G^{B C}+M_{2}^{B C}, V_{M}^{B C \prime}=M_{1}^{B C}$,
$V_{R}^{B C}=R_{1}^{B C} G^{B C}+R_{2}^{B C}, V_{R}^{B C^{\prime}}=R_{1}^{B C}$.

Where $M_{1}^{B C}, M_{2}^{B C}, R_{1}^{B C}, R_{2}^{B C}$ are parameters. Substituting (77)-(78) and (80)-(84) into the HJB equations and simultaneous equations (85) and (86), we obtain:

$$
\begin{aligned}
& 2 \mathrm{~b} \theta\left(2 \mathrm{~b}^{2}-2\right)(\mathrm{b} \theta+(1-\theta))-2 \mathrm{~b}^{2}((1-\theta)+\mathrm{b} \theta)^{2} \\
& +(b \theta+(1-\theta))\left((1-\theta)\left(3 b^{2}-1\right)+2 b \theta\right) \\
& +2 b\left(2 b^{2}-2\right)(1-\theta)(b(1-\theta)+\theta) \\
& +2 \mathrm{~b}(\mathrm{~b}(1-\theta)+\theta)(\mathrm{b} \theta+(1-\theta)) \\
& -\left(b^{2}(1-\theta)+b \theta\right)\left((1-\theta)\left(3 b^{2}-1\right)+2 b \theta\right) \\
& =2 \mathrm{~b}\left(2 \mathrm{~b}^{2}-2\right)^{2}(\mathrm{r}+\delta) \mathrm{M}_{1}^{\mathrm{BC}} \text {, } \\
& -\left(\mathrm{R}_{1}^{\mathrm{BC}}\right)^{2} \gamma^{2}\left(1-\eta_{\mathrm{M}}^{\mathrm{BC}}\right)^{2}\left(1-\eta_{\mathrm{R}}^{\mathrm{BC}}\right)+2 \mathrm{M}_{1}^{\mathrm{BC}} \mathrm{R}_{1}^{\mathrm{BC}} \epsilon^{2}\left(\eta_{\mathrm{R}}^{\mathrm{BC}}\right)^{2}\left(1-\eta_{\mathrm{M}}^{\mathrm{BC}}\right)-\left(\eta_{\mathrm{R}}^{\mathrm{BC}}\right)^{2} \eta_{\mathrm{M}}^{\mathrm{BC}}\left(\mathrm{R}_{1}^{\mathrm{BC}}\right)^{2} \epsilon^{2} \\
& 4 b^{2}\left(2 b^{2}-2\right)^{2}(r+\delta) R_{1}^{B C} \\
& =4 \mathrm{~b}\left(\mathrm{~b}^{2}-1\right)\left(\mathrm{b}^{2}-1\right)(1-\theta)^{2} \\
& -b(1-\theta)\left(b^{2}-1\right)\left(2 b \theta+(1-\theta)\left(3 b^{2}-1\right)\right) \\
& +2 b(1-\theta)\left(b^{2}-1\right)(b \theta+(1-\theta)) \text {, } \\
& \left(\mathrm{R}_{1}^{\mathrm{BC}}\right)^{2} \epsilon^{2} \eta_{\mathrm{R}}^{\mathrm{BC}}+\left(\mathrm{R}_{1}^{\mathrm{BC}}\right)^{2} \gamma^{2}\left(1-\eta_{\mathrm{M}}^{\mathrm{BC}}\right)=2 \mathrm{rR}_{2}^{\mathrm{BC}} \eta_{\mathrm{R}}^{\mathrm{BC}}\left(1-\eta_{\mathrm{M}}^{\mathrm{BC}}\right) \text {. }
\end{aligned}
$$$$
+2 \mathrm{M}_{1}^{\mathrm{BC}} \mathrm{R}_{1}^{\mathrm{BC}} \gamma^{2} \eta_{\mathrm{R}}^{\mathrm{BC}}\left(1-\eta_{\mathrm{M}}^{\mathrm{BC}}\right)^{2}=2 \mathrm{r}\left(1-\eta_{\mathrm{M}}^{\mathrm{BC}}\right)^{2}\left(\eta_{\mathrm{R}}^{\mathrm{BC}}\right)^{2} \mathrm{M}_{2}^{\mathrm{BC}},
$$ 
where the parameters $M_{1}^{B C}, M_{2}^{B C}, R_{1}^{B C}, R_{2}^{B C}$ can be easily obtained through simultaneous equations (87) - (90). Then, inserting $M_{1}^{B C}$ and $R_{1}^{B C}$ into (77), (78), (82) and (83), we obtain

$$
\begin{aligned}
& A_{M}^{B C}=\frac{\gamma(-1+\theta)^{2}}{16 b(r+\delta)}+\frac{\gamma\left(1+b^{2}(1-\theta)^{2}+4 b \theta(1-\theta)+2 \theta\left(b^{2}-1\right)+\theta^{2}\right)}{4 b\left(b^{2}-1\right)(r+\delta)}, \\
& A_{R}^{B C}=\frac{\epsilon(-1+\theta)^{2}}{16 b(r+\delta)}+\frac{\epsilon\left(1+b^{2}(1-\theta)^{2}+4 b \theta(1-\theta)+2 \theta\left(b^{2}-1\right)+\theta^{2}\right)}{4 b\left(b^{2}-1\right)(r+\delta)}, \\
& \eta_{M}^{B C}=\frac{5+3 b^{2}-10 \theta+16 b \theta-6 b^{2} \theta+5 \theta^{2}-16 b \theta^{2}+11 b^{2} \theta^{2}}{3+5 b^{2}-6 \theta+16 b \theta-10 b^{2} \theta+3 \theta^{2}-16 b \theta^{2}+13 b^{2} \theta^{2}} \\
& \eta_{R}^{B C}=\frac{2\left(-1+b^{2}\right)(-1+\theta)^{2}}{3+5 b^{2}-6 \theta+16 b \theta-10 b^{2} \theta+3 \theta^{2}-16 b \theta^{2}+13 b^{2} \theta^{2}} .
\end{aligned}
$$

\section{Acknowledgement}

This research was partially supported by a research grant of Korea Research Foundation of 2017 (No. NRF-2016R1A2B1015318).

\section{References}

[1] S. Jørgensen, S. P. Sigué and G. Zaccour, "Dynamic cooperative advertising in a channel”, Journal of Retailing, vol. 76, (2000), pp. 71-92, https://doi.org/10.1016/S0022-4359(99)00024-X.

[2] M. G. Nagler, "An exploratory analysis of the determinants of cooperative advertising participation rates", Marketing Letters, vol. 117, (2006), pp. 91-102, https://doi.org/10.1007/s11002-006-4387-0.

[3] A. R. Borrell, "The changing face of co-op programs", (2015), Available at: https://www.netsertive.com/.

[4] R. Yan, P. Guo, J. Wang and N. Amrouche, "Product distribution and coordination strategies in a multichannel context", Journal of Retailing and Consumer Services, vol. 18, (2001), pp. 19-26, https://doi.org/10.1016/j.jretconser.2010.09.001.

[5] M. Nerlove and K. J. Arrow, "Optimal advertising policy under dynamic conditions", Economica, vol. 39, (1962), pp. 129-142, https://doi.org/10.2307/2551549.

[6] P. D. Berger, "Vertical cooperative advertising ventures", Journal of Marketing Research, vol. 9, (1972), pp. 309-312, https://doi.org/10.2307/3149542.

[7] P. K. Chintagunta and D. Jain, "A Dynamic Model of Channel Member Strategies for Marketing Expenditures”, Marketing Science, vol. 11, (1992), pp. 117-134, https://doi.org/10.1287/mksc.11.2.168.

[8] S. Jørgensen, S. P. Sigué and G. Zaccour, "Stackelberg leadership in a marketing channel", International Game Theory Review, https://doi.org/10.1142/S0219198901000282, vol. 3, (2001), pp. 13-26.

[9] R. P. Dant and P. D. Berger, "Modeling Cooperative Advertising Decisions in Franchising", Journal of the Operational Research Society, vol. 49, (1996), pp. 1120-1136, https://doi.org/10.1057/jors.1996.141.

[10] M. Bergen and G. John, "Understanding Cooperative Advertising Participation Rates in Conventional Channels", Journal of Marketing Research, vol. 46, (1997), pp. 357-369, https://doi.org/10.2307/3151898.

[11] S. Y. Kim and R. Staelin, "Manufacturer Allowances and Retailer Pass-Through Rates in a Competitive Environment", Marketing Science, vol. 18, (1999), pp. 59-76, https://doi.org/10.1287/mksc.18.1.59.

[12] Z. M. Huang and S. X. Li, "Co-op advertising models in a manufacturing-retailing supply chain: a game theory approach", European Journal of Operational Research, vol. 135, (2011), pp. 81-98, https://doi.org/10.1016/S0377-2217(00)00327-1.

[13] M. M. SeyedEsfahani, M. Biazaran and M. Gharakhani, "A game theoretic approach to coordinate pricing and vertical co-op advertising in manufacturer-retailer supply chains", European Journal of Operational Research, vol. 211, (2011), pp. 263-273, https://doi.org/10.1016/j.ejor.2010.11.014.

[14] S. Jørgensen, S. Taboubi and G. Zaccour, "Retail promotions with negative brand image effects: is cooperation possible?", European Journal of Operational Research, vol. 150, (2003), pp. 395-405, https://doi.org/10.1016/S0377-2217(02)00641-0.

[15] S. Karray and G. Zaccour, "A differential game of advertising for national and store brands", In Haurie, A., \& Zaccour, G (Eds.), Dynamic Games: Theory and Applications, New York, USA: Springer, (2005), pp. 213-229. 
[16] X. L. He, A. Krishnamoorthy, A. Prasad and S. P. Sethi, "Retail competition and cooperative advertising", Operations Research Letters, https://doi.org/10.1016/j.orl.2010.10.006, vol. 39, (2011), pp. 11-16.

[17] P. De Giovanni and M. Roselli, "Overcoming the drawbacks of a revenue-sharing contract through a support program", Annals of Operations Research, vol. 196, (2012), pp. 201-222, https://doi.org/10.1007/s10479-012-1113-6.

[18] S. Taboubi and G. Zaccour, "Impact of Retailer's myopia on channel's strategies'”, In Zaccour, G (Eds.), Optimal control and differential games. Essays in honour of Steffen Jørgensen, New York, USA: Springer, (2002), pp. 179-192.

[19] A. Chutani and S. P. Sethi, "Optimal advertising and pricing in a dynamic durable goods supply chain", Journal of Optimization Theory Applications, vol. 154, (2012), pp. 615-643, https://doi.org/10.1007\%2Fs10957-012-0034-5.

[20] P. De Giovanni, "Environmental collaboration in a closed-loop supply chain with a reverse revenue sharing contract", Annals of Operations Research, vol. 220, (2014), pp. 135-157, https://doi.org/10.1007/s10479-011-0912-5.

[21] N. Singh and X. Vives, "Price and quantity competition in a differentiated duopoly", Rand Journal of Economics, vol. 15, (1984), pp. 546-554, https://doi.org/10.2307/2555525. 